\title{
The current status of Open Access in biomedical field: the comparison of countries relating to the impact of national policies
}

\section{Mamiko Matsubayashi}

Graduate School of Library, Information and Media studies, University of

Tsukuba, 1-2 Kasuga, Tsukuba City, Ibaraki 305-8550,

Japan mamiko@slis.tsukuba.ac.jp

\section{Keiko Kurata}

School of Library and Information Science, Keio University, 2-15-45 Mita,

Minato-ku, Tokyo 108-8345, Japan keiko@slis.keio.ac.jp

\section{Yukiko Sakai}

Shinanomachi Media Center, Keio University, 35 Shinanomachi, Shinjuku-ku

Tokyo 160-8582, Japan yukiko@lib.keio.ac.jp

\section{Tomoko Morioka}

Kunitachi College of Music Library, Kunitachi College of Music, 5-5-1

Kashiwa-cho, Tachikawa City, Tokyo, 190-8520, Japan QYB00077@nifty.com

\section{Shinya Kato}

Yamagata University Library, Yamagata University, 1-4-12 Kojirakawa-machi, Yamagata-shi, Yamagata, 990-8560, Japan skato@jm.kj.yamagata-u.ac.jp

\section{Shinji Mine}

Graduate School of Library and Information Science, Keio University, 2-15-45 Mita, Minato-ku, Tokyo 108-8345, Japan mine@slis.keio.ac.jp

Shuichi Ueda

School of Library and Information Science, Keio University, 2-15-45 Mita, Minato-ku, Tokyo 108-8345, Japan ueda@slis.keio.ac.jp 
The purpose of the article is to show the current status of Open Access (OA) in biomedical field, and compare some countries such as the U.S., the U.K. and Japan in terms of the $O A$ situation. There are controversies about the definition of $O A$. After examining the requirements about $O A$, we recognized $O A$ as the situation in which researchers could read the full text of articles in unrestricted way. In order to investigate the current situation of $O A, 4,756$ articles were sampled randomly from articles published between January and September in 2005 and indexed in PubMed. The main results are as follows: 1) The rate of OA articles was $25 \%$, and $75 \%$ of all the articles were available online including electronic subscription journal articles. 2) The means of $O A$ was classified into five types. Among them, the rate of OA articles by "OA and Hybrid OA journals" was overwhelming (more than $70 \%$ ), and that of PMC was $26.2 \%$. The rates of OA articles by "institutional repositories" and "authors' personal sites" were considerably low ( $6.0 \%$ and $4.9 \%$ respectively). 3) When comparing the rates of

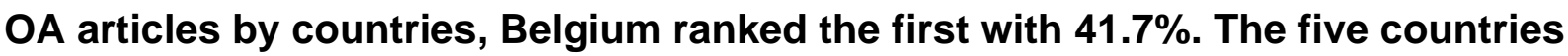
indicated more than $30 \%$ in OA articles: Canada and India (38.7\%), Brazil (36.4\%), Australia (30.8\%), and the U.S. (30.7\%). Each country was different in the means of OA. 4) We explored the rates of OA for two groups; one group consists of articles published in journals with IF, and the other consists of articles published in journals without IF. The rate of OA for the group of articles in journals with IF is $\mathbf{2 0 . 6 \%}$, and that of articles in journals without IF is $\mathbf{3 0 . 8 \%}$.

Introduction 
Open Access movement and its concept have attracted a lot of attention in scholarly communication for the last several years. Although different attitudes have been showed in the discussion on Open Access, few empirical studies have been made at the time of writing. The purpose of this study is to clarify the current status of Open Access in biomedical field as of 2005.

The study focuses on biomedical field, because one of the authors' interests was how the National Institutes of Health public access policy (NIH, 2005) has affected scholarly communication. This policy has attracted considerable attention. Some expect that the policy may not encourage Open Access, since the latest policy has toned down from the first proposal under great pressure from commercial publishers and academic societies. The policy allowed a longer embargo from six months to one year and registration to PubMed Central (PMC) was not required anymore. On the other hand, others expect that the NIH policy may have a great impact, because the policy was stated by NIH which is one of the largest research-funding agencies in the world.

We would like to pay attention to the current status of Open Access, for instance which information could be accessible, and how it could be accessed. Moreover this study will compare the U.S., the U.K., Japan, and some other countries in terms of the current situation of Open Access. It is because national policies such as NIH as mentioned above may have had a different effect on Open Access situation in each country.

The next sections will discuss the definition and means of Open Access and the current situation of Open Access movement in each country as background of the study and at the end, literature will be reviewed.

\section{The definition of "Open Access"}

There are many kinds of definitions of "Open Access (hereafter OA)." Budapest Open Access Initiative (BOAI) defined $\mathrm{OA}$ as "the world-wide electronic distribution of the peer-reviewed journal literature and completely free and unrestricted access to it" (BOAl, 2002). Suber (2004) also gave a similar definition: "open access (OA) literature is digital, online, free of charge, and free of most copyright and licensing restrictions." The requirements of $\mathrm{OA}$ derived from those definitions are as follows:

1. Online or electronic version

$O A$ is based on the assumption that literature (scholarly information) is online or electronic version. OA has been realized only after the diffusion of the Internet as 
computer network infrastructure and a mechanism of the electronic distribution of scholarly information.

2. Unrestricted access which includes free access

$O A$ is frequently recognized as free of charge access; however, the most important principle is unrestricted access for researchers. This is an attitude that while the author's basic rights are protected, literature should be freely available for public use. The copyright of academic journal articles usually belongs to commercial publishers or academic societies. There are many concerns with their attitudes to $\mathrm{OA}$, namely how far they could allow the authors to make their articles available freely. One issue is which version (e.g. the author's final draft (not PDF) or PDF files of electronic journal articles supplied by the publisher) could be $O A$. Another is whether PDF files could be distributed by the authors. Yet another issue is embargo. Harnad (2005) emphasizes that journal articles should be publicly available right after the publication. He pointed out that free of charge access with embargo is not OA.

3. Scholarly information

The object of $\mathrm{OA}$ is at least scholarly information which is considered in public domain. As it was said in BOAl, researchers have the "Old tradition" - they wish their research results to be widely distributed free of charge. The concept of OA relies on this tradition. Peer-reviewed journal articles must constitute the core of OA, while the object may not be restricted to the peer-reviewed articles.

This study defines $\mathrm{OA}$ as the situation in which an article can be obtained online by researchers at no charge without complicated procedure when they hope to use it ; the study deals with any kind of literature in biomedical field as the subject of the investigation (not restricting the object to the peer-reviewed articles).

\section{Means of OA}

Two means of OA are self-archiving and Open Access journals (BOAI, 2002). In self-archiving, an author makes his/her articles publicly available outside of an existing publishing route (mainly scholarly journals by commercial publishers or academic societies). For example, researchers can post an article full text on their own websites. Other types of self-archiving are subject-based repositories or institutional repositories. arXiv is a spontaneous subject-based repository that collects and supplies electronic preprint of journal articles in the field of physics. Institutional repositories (IR) collect and supply research achievements of affiliated researchers. Registry of Open Access Repositories (ROAR) shows the overview of more than 600 institutional repositories in the world. U.K. Science and Technology Committee recommends IR as the most effective way 
of OA (House of Commons Science and Technology Committee, 2004).

NIH's PMC and UK PubMed Central (UK PMC) which is to be launched in 2006 may also be considered as subject-based repositories as a self-archiving mechanism; however, the nature is different from e-print archives such as arXiv. PMC and UK PMC are official digital archives which may force the registration of articles on the authors who receive funding from governmental institutions, while arXiv is a voluntary information exchange mechanism among researchers. Because of one-year embargo, Harnad (2005) calls PMC "Back access" instead of "Open access".

The second means of $O A$ is submitting an article to an Open Access journal. Controversies abound about what journal could be called OA. The points are: types of articles, embargo, and a cost model. DOAJ (Directory of Open Access Journals) maintained by University of Lund, Sweden defines Open Access journals as journals that do not charge readers or their institutions for access and allow the users to "read, download, copy, distribute, print, search, or link to the full texts of these articles"; however, some of journals on the directory DOAJ do not satisfy the conditions above. This will be discussed in detail in the review.

The more complex situation is represented by OA hybrid journals which make an article in traditional subscription journals publicly available only if the author pays the fee (SPARC open access newsletter, 2006).

This study has attempted to reveal the means of OA : self-archiving or Open Access journals; the detail of self-archiving or Open Access journals.

\section{Open Access movement in each country}

This study will examine the current situation of OA in each country. Although the current scholarly communication still depends on a traditional model consisting of commercial publishers, academic societies and academic libraries, new stakeholders have been involved in OA movement. For example, governmental agencies or research-funding institutions have given a statement to encourage $O A$ and suggest a concrete support system. In order to reveal the influence of those statements considered as a kind of their policies, the results of the investigation on $\mathrm{OA}$ will be examined by authors' affiliations by countries.

This section will introduce policies in the U.S., the U.K. and other countries, and Japan. The position of Japan must be distinguished, because Japan hasn't had a specific national policy on OA compared to the U.S. and the U.K. where national governmental agencies 
have stated policies.

The U.S.

In the U.S., NIH's public access policy has been in effect since May 2005. Research publications based on NIH fund have been requested to be posted to PubMed Central within one year. Because the embargo could be longer (i.e. one year instead of six months), an immediate OA hasn't been encouraged. However, PMC works as an official digital archive. Also institutional repositories work for OA in many universities. For example, University of California has organized a committee to discuss scholarly communication issues and support $\mathrm{OA}$ movement as a higher educational institution. There have been some other universities (e.g. Queensland University of Technology, Swiss Federal Institute of Technology in Zurich) which force the registration of the affiliated researchers' publications into their IR (Institutional Self-Archiving Policy Registry).

\section{The U.K. and other countries}

In the U.K., Science and Technology Committee published "Scientific publications free for all?" in 2004 and recommended institutional repositories in further and higher educational institutions to support Open Access. Since then, JISC (The Joint Information Systems Committee), an advisory committee on the use of information technology for learning, teaching, and research, has supported institutional repositories in some aspects. Wellcome Trust, the largest private research-funding agency, first expressed its support to $O A$ in 2003, and from 2005 has forced OA on research publications based on Wellcome Trust funded research through PMC or UK PMC. RCUK (Research Councils UK) also insists that their funded research publications should be $O A$.

In most European countries such as France, Germany, Sweden and Finland, in addition to the U.S. and the U.K., OA policy has been claimed by governmental agencies or committees. The other countries outside of Europe which support $O A$ are India and Brazil.

\section{Japan}

Differently from the case in the U.S., the U.K., and many European countries, a major support for scholarly communication in Japan has been set up for electronic journal publishing by academic societies rather than $\mathrm{OA}$. The academic societies are expected to use J-STAGE, which is a common electronic journals platform operated by JST (Japan 
Science and Technology Agency), an independent Administrative Institution which was originally a governmental agency. Academic societies can make their journal articles online free of charge (using J-STAGE), if they prepare data (i.e. PDF file, metadata and citation data). Japan ranks high among other countries in comparison of the number of OA journals, according to the OA journal list in DOAJ. Most journal titles listed in the directory are on J-STAGE. It is speculated that many academic societies are operated by the income from print journals and make their articles online using J-STAGE free of charge. This operation results in $\mathrm{OA}$ through the governmental common platform.

Council for Science and Technology Policy released "Science and Technology Basic Plan 2006-2011" in December 2005. The plan only emphasizes the expansion of an infrastructure for science and technology and shows no intention of promoting $\mathrm{OA}$. The plan only mentions about public-funded research articles: "the authors are expected to make their articles publicly available with relevant embargo when their research results based on public-funded research are published" (Council for Science and Technology Policy, 2005).

Another related proposal was posted in March 2006 by a committee which deals with issues in scholarly communication. The committee was organized by the Ministry of Education, Culture, Sports, Science, and Technology. The report of the committee covers promotion of institutional repositories by academic libraries and a support for academic societies for electronic journals; however, it doesn't directly support Open Access. Institutional repositories have been already implemented or in the test phase in about ten universities in Japan with a support from the National Institute of Informatics.

\section{Literature review}

The situation of OA has been investigated from three aspects: 1) the rate of OA articles, 2) the quantity and the nature of OA journals, 3 ) the situation of OA movement among researchers.

\section{The rate of $O A$ articles}

Most of quantitative analysis of OA articles focused on the impact of OA articles on Non-OA articles and did not show the absolute rate of the OA articles. Lawrence (2001) who reported the impact of $O A$ for the first time is a typical example.

The rate of $O A$ articles varies with academic fields. Hajjem et al. (2005) reported the current situation and the impact of $O A$ based on the analysis of over 140,000 article 
records in 10 academic fields (i.e. biology, psychology, sociology, health, political science, economics, education, law, business, and management) published between 1992 and 2003. The records were derived from Thomson Scientific's Web of Science. The rates of OA articles were $5 \sim 16 \%$ as a whole, $15 \%$ in biology and $6 \%$ in health. Harnad and Brody (2004) showed rates in physics: 10\% in average between 1992 and 2001; 18\% in 2001. The highest rate was showed in nuclear and particle physics as over $40 \%$ in 1996 and $48 \%$ in 2001 . This specific field has been famous for its higher number of registrations in arXiv from early stage. Kurtz et al. (2005) found that $70 \%$ of articles in Astrophysical Journal, a core journal in the field, published in $\mathbf{2 0 0 3}$ had been registered in arXiv. Anteleman (2004) compared OA in four kinds of academic fields (i.e. philosophy, political science, electrical and electronic engineering, and mathematics) analyzing articles published in 2001 and 2002 in ten journals in each field. The rate varies from $17 \%$ in philosophy to $69 \%$ in mathematics.

Hajjem et al. (2005) also showed the average rate by countries: $13 \%$ in the U.S., $10 \%$ in the U.K., $7 \%$ in Japan, $7 \%$ in Germany, and 13\% in France.

Means of Open Access were investigated by Antelman (2004). Except in mathematics, self-archiving is the most popular means of Open Access such as $36 \%$ in philosophy, over $20 \%$ in political science and electrical and electronic engineering. In mathematics, subject-based depository is much more popular (30\%) than self-archiving (15\%).

Kurtz et al. (2005) suggested that some selection policy may work whether articles could be registered in arXiv. Miyairi (2005) pointed out that the results of the investigations on OA impact may be biased toward qualified articles because they tended to deal with arXiv, Web of Science, and sampling from prestigious journals. She designated as a problem whether or not researchers could retrieve OA articles.

\section{The quantity and the nature of $O A$ journals}

The increasing number of OA journals may be found in DOAJ and Ulrich's directory. For example, DOAJ lists more than 2,000 journals as of February 3, 2006. According to Morris's analysis of 1,213 journals in the directory, $14 \%$ had problems (e.g. partly inaccessible, no articles were published after 2003) against BOAl definition for OA (Morris, 2006). Other findings of Morris were: many journals are the former subscription print journals; the oldest OA articles were published mostly in 2000; the average number of articles published per year was 42, while the mode value was 10. 
Swan and Brown (2005) conducted a survey among 25,000 authors derived from Web of Science in 2004 and received 1,296 respondents. 49\% of the authors had self-archived their article in the last three years. The means of OA for peer-reviewed articles were personal websites (31\%), institutional repositories (25\%) and subject-based open archives (15\%).

\section{Research design and method}

Research questions

This study is an attempt to show the current situation of OA from an aspect of whether articles are accessible for researchers unrestrictedly. In order to include all kinds of journal articles in biomedical field, PubMed was used instead of Web of Science to derive subject articles. The articles were searched using search engines and other databases to know if their full text files are available.

The hypotheses led by the existing studies were:

1. The rate of $O A$ is higher than in social sciences, but lower than in physics. It is estimated that the rate is around $20 \%$, since it could increase from $15 \%$ in biology in 2003.

2. The most popular means of $O A$ is PMC because of the influence of NIH's public access policy.

3. The means may be different from country to country depending on national $\mathrm{OA}$ policies.

4. The rate of OA in Japan is lower than in other countries; J-STAGE is the most frequently used platform in OA in Japan.

5. Articles published in prestigious journals show higher rate of $O A$.

\section{Method}

\section{Sampling}

In order to generate a target sample for analysis, we chose PubMed, which provides a broad coverage and is the most popular database in biomedical field. We took a random sample using page number from all the articles published between January and September in 2005 and indexed in PubMed. "Editorial" articles and articles without author's name or titles, however, were excluded. 


\section{Procedure}

We specified whether a full text (FT) of the articles is freely available as an OA article searching PubMed, PubMed Central (PMC), and search engines such as Google (Fig.1).

First, PubMed was searched to verify the bibliographic data. Second, we searched PubMed Central, Google Scholar, and Google in this order until the FT for the article was found PMC was searched by title or title plus author's name; when the FT was not found in PMC, Google Scholar was searched by title plus author's name, title only or author's name only; only when the FT was not found both in PMC and Google Scholar, Google was searched in the same manner as Google Scholar search. Lastly, the FT of all the articles was searched against OAl-ster by title or title and author's name. The title was searched as a phrase against all the databases. The author search was done by a sir name of the first author only. We examined the first 20 only in the result list. When the full text was found, the URL was recorded with a code that represents four categories as follows:

1: OA, 2: Restricted OA (e.g. need for registration), 3: Non OA (subscription electronic journal page), 0: Not online available.

\section{Other basic data}

In order to compare the current situations of OA by countries, "country" was assigned for target articles according to the affiliation of the first author. Impact Factors of journals in which target articles were published were collected from JCR 2004. 


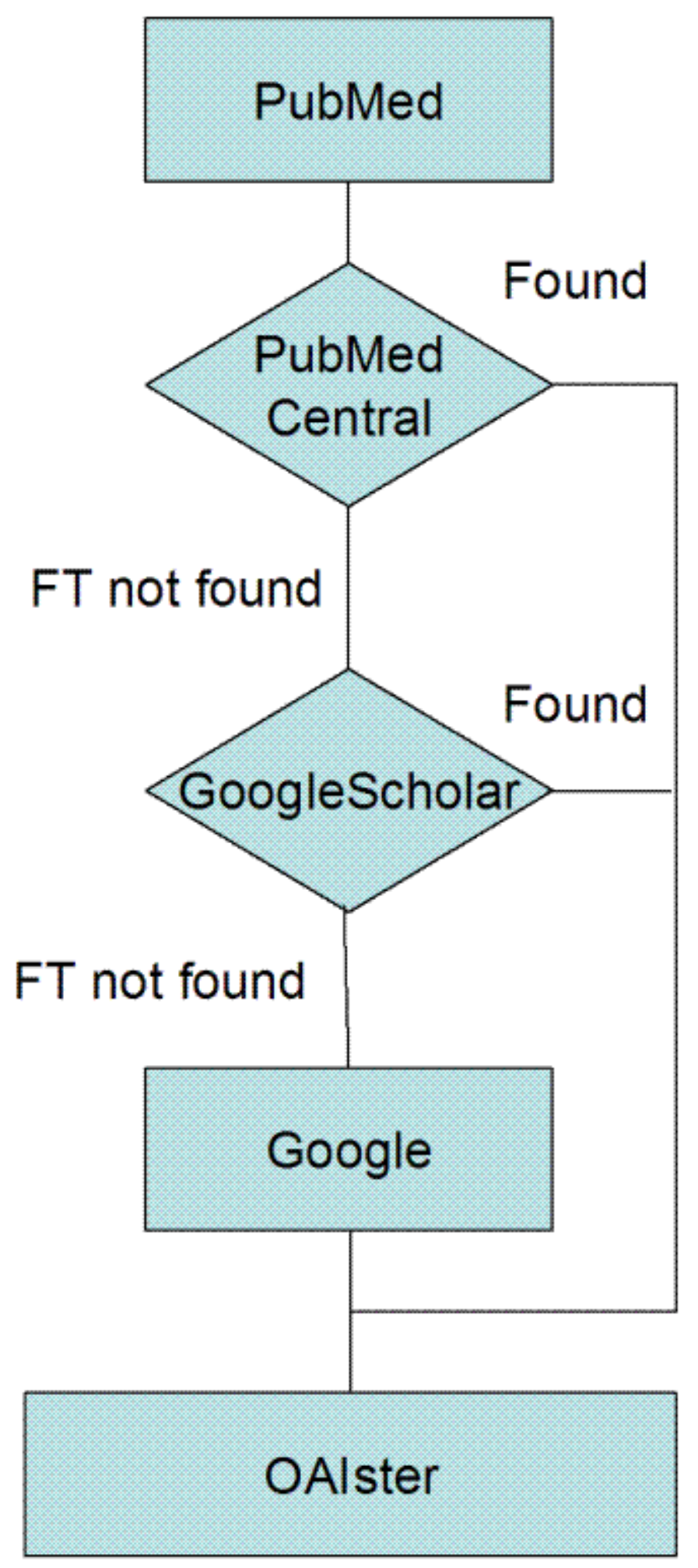

\section{PIDSearch}

Title or

Title plus author Search

Title plus author, title only, or author only Search

Title plus author, title plus journal name, or title only Search

Title or

Title plus author Search

Figure 1. Search procedure of the investigation 
Rates and means of $O A$

Rates of OA

Table1 shows each percentage of "OA" articles, "restricted OA" articles, "electronic subscription journal" articles, and "not online available" articles.

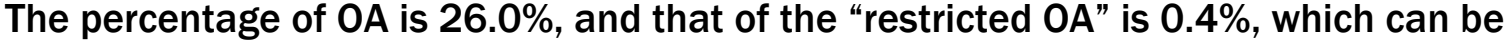
accessed free, but is in need of registration. $53.9 \%$ of all the articles were available through the electronic subscription journals, and $19.7 \%$ were not available online.

Table 1. The rates of $O A$

\begin{tabular}{|l|l|c|c|}
\hline \multirow{3}{*}{ Online available } & OA & 1235 & $26.0 \%$ \\
\cline { 2 - 3 } & Restricted OA & 21 & $0.4 \%$ \\
\cline { 2 - 3 } & Not OA & 2565 & $53.9 \%$ \\
\hline Not online available & 935 & $19.7 \%$ \\
\hline Total & 4756 & $100.0 \%$ \\
\hline
\end{tabular}

About a quarter of articles in biomedical field were available as OA articles. This figure is higher than in social sciences, but lower than in physics, as stated in the previous section. In comparison with the results of investigation by Hajjem et al. (2005) that the rate of OA in biology in 2003 was $15 \%$, the growth rates of OA may be considerable.

\section{Means of $O A$}

The Means of OA were classified into five types: 1) PMC, 2) OA or Hybrid OA journals, 3) institutional repositories, 4) authors' personal sites, 5) Portal sites or journals platform in which governmental agency, academic association, and private companies provide many electronic journals. There are $\mathrm{OA}$ articles available by multiple means.

Fig. 2 shows the percentage of $O A$ articles available by five means. The percentage of $O A$ articles available from "OA or Hybrid OA journals", in which OA can be provided by the journals, not authors, is overwhelming (72.6\%). That of PMC and "portal sites or platforms for multiple electronic journals" followed with $26.2 \%$ and $17.6 \%$. In "portal sites or platforms for multiple electronic journals", J-STAGE by JST in Japan and the Scientific Electronic Library Online (SciELO) in Brazil were included. In contrast, the rates of OA articles available from typical self-archiving, institutional repositories and authors' personal sites, were considerably low (6.0\% and $4.9 \%$ respectively). 


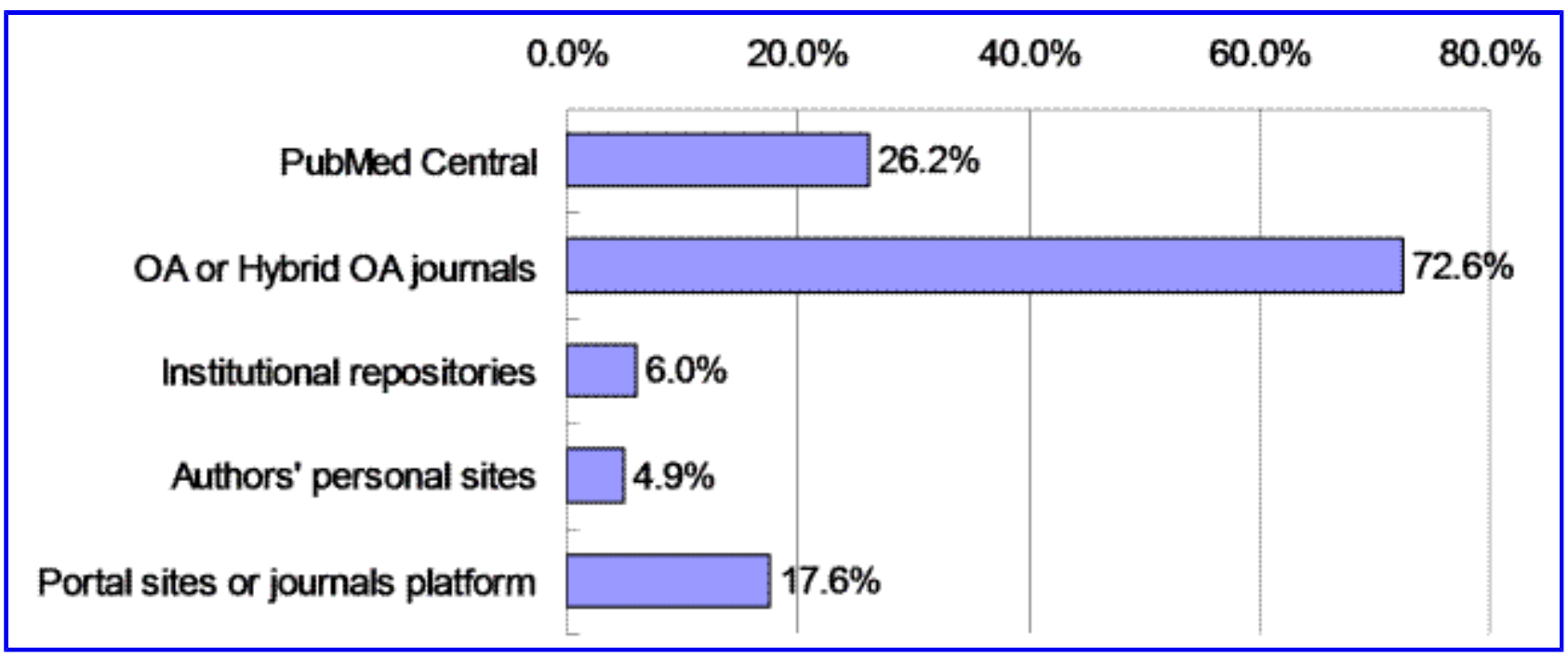

Figure 2. The means of OA (multiple answers)

The rate of $\mathrm{OA}$ through $\mathrm{PMC}$ was $26.2 \%$, which is much higher than that through the other means except for "OA or Hybrid OA journals". However 93\% of the OA articles through PMC were also OA in "OA journals". It showed that most of the articles in PMC have been through OA journals by BioMed Central and so on. Opposite to PMC, only $12 \%$ of the OA articles in "institutional repositories" were also OA through "OA or Hybrid OA journals". Institutional repositories may realize OA of the articles which could not be OA through "OA or Hybrid OA journals".

The current situation of OA in each country Comparison by countries

In this section, we calculated 3,783 articles excluding articles without author's affiliation, instead of all the samples. Table 2 indicates the current situations of the top 20 countries which published the articles.

The number of articles published in the U.S. is the largest and occupies about $33 \%$. The U.K., Japan, Germany, China, Canada, Italy follow.

Among the 20 countries, Belgium marked the highest rate of OA articles with $41.7 \%$, followed by Canada and Sweden (38.7\%), Brazil (36.4\%). As these countries (except for Canada), however, published less than $2 \%$ of the total samples, the small sample size may affect high rates of OA. The rate of OA articles in Canada was the highest (38.7\%) within top 8 countries in the number of articles and that of the U.S. as the second country, was over $30 \%$ (30.7\%). Four countries indicated more than $20 \%$ in OA articles: the U.K. (22.8\%), France (22.1\%), Italy (20.4\%), Japan (20.2\%). 
Table 2. The current $O A$ situations of the top 20 countries

\begin{tabular}{|c|c|c|}
\hline Country & Total & The rate of OA \\
\hline USA & 1261(33.3\%) & $30.7 \%$ \\
\hline UK & $320(8.5 \%)$ & $22.8 \%$ \\
\hline Japan & $243(6.4 \%)$ & $20.2 \%$ \\
\hline Germany & $219(5.8 \%)$ & $18.3 \%$ \\
\hline China & $157(4.2 \%)$ & $17.8 \%$ \\
\hline Canada & $150(4.0 \%)$ & $38.7 \%$ \\
\hline Italy & $137(3.6 \%)$ & $20.4 \%$ \\
\hline France & 131(3.5\%) & $22.1 \%$ \\
\hline Australia & $107(2.8 \%)$ & $30.8 \%$ \\
\hline Netherlands & $91(2.4 \%)$ & $26.4 \%$ \\
\hline Spain & $82(2.2 \%)$ & $24.4 \%$ \\
\hline Sweden & $70(1.9 \%)$ & $28.6 \%$ \\
\hline India & $62(1.6 \%)$ & $38.7 \%$ \\
\hline Switzerland & $61(1.6 \%)$ & $18.0 \%$ \\
\hline Brazil & $55(1.5 \%)$ & $36.4 \%$ \\
\hline Turkey & $53(1.4 \%)$ & $24.5 \%$ \\
\hline Poland & $42(1.1 \%)$ & $23.8 \%$ \\
\hline Belgium & $36(1.0 \%)$ & $41.7 \%$ \\
\hline Korea, Republic of & $35(0.9 \%)$ & $22.9 \%$ \\
\hline Taiwan & $31(0.8 \%)$ & $19.4 \%$ \\
\hline
\end{tabular}

Top 20 countries (except for Brazil) marked the high percentage of OA available from "OA or Hybrid OA journals". Comparing the means of OA in the U.S., the U.K. and Japan, each country marked the high percentage of OA available from "OA or Hybrid OA journals". Other rates of means, however, varied with the U.S., the U.K. and Japan. In the U.S. and the U.K., the rate of OA available from "OA or Hybrid OA journals" is extremely high (70 or $80 \%$ ), and that of PMC is $30 \%$, which is a little higher than the average. The two countries are different in the other means of OA. In the U.S., OA articles available from "portal sites" occupied $15 \%$, which is comparatively high. In the U.K., the rate of OA available from "authors' personal sites" is $11.0 \%$. The examination showed that Japan differed in its means of $O A$, so in the next section the characteristic of OA in Japan will be described. 
Only $6.4 \%$ of all the articles indexed in PubMed were what Japan published. This figure is quite lower in comparison with the result by Adachi et al. (2003) which indicates that journal articles by Japanese researchers in STM fields accounted for about $12 \%$.

The rate of $\mathrm{OA}$ articles is $20.2 \%$. It ranks sixteenth among top 20 countries; however, it is by no means high. Japan showed characteristic patterns although the rate of OA from "OA or Hybrid OA journals" is the highest among the means of OA, which is the same trend in the U.S. and the U.K. On the one hand, "Journals platform" (J-STAGE of JST) and "OA or Hybrid OA journals" accounted for $40.8 \%, 57.1 \%$; on the other, PMC (10.2\%), "authors' personal sites" (6.1\%) and "institutional repositories" (2.0\%) were seldom used for the means of OA. This pattern is different from that of the U.S. or European countries, and is similar to that found in Brazil, India, and so on.

The rate of OA by journals with/without Impact Factor (IF)

When investigating the Impact Factor (IF) for journals in which all the sample articles $(4,756)$ were published, half of the articles (52.4\%) published in journals did not have IF.

The rate of $O A$ for the group of articles in journals with IF is $20.6 \%$, and that of articles in journals without IF is $\mathbf{3 0 . 8 \%}$. The rate of OA in journals without IF is a bit higher, and the rates of other articles than $\mathrm{OA}$ articles are quite different. Among articles in journals with IF, the rate of "not online available" articles is only $5.6 \%$ and that of "electronic subscription journal" articles is $73.3 \%$. By contraries, the rate of "not online available" articles is $32.4 \%$ and that of "electronic subscription journal" articles is $36.3 \%$, among articles in journals without IF.

Articles in journals with IF which should be major journals in the field, can be mostly available online (it is an assumption for $O A$ ), but the rate of making $O A$ is not so high. In comparison, considerable parts of articles in journals without IF are not available online, but the rate of $\mathrm{OA}$ is higher than that of articles in journals with IF.

We compared the means of $\mathrm{OA}$ in two journal groups: one consisted of journals with IF and the other without IF. The rates of OA through PMC are $11.1 \%$ and $35.4 \%$ for articles in journals with IF and without IF respectively. A little less than $90 \%$ of articles registered in PMC were published in journals without IF. On the other hand, the rates of "authors' personal sites" are $10.5 \%$ for journals with IF and only $1.7 \%$ for journals without IF. More than $90 \%$ of OA articles through "authors' personal sites" were published in journals with IF. 


\section{Discussion}

We examined the results of OA situation in biomedical field from two points of view: 1) the characteristics of $O A$ articles, 2) national policies relating to scholarly communication in each country.

\section{The characteristics of OA article}

We selected PubMed, not Web of Science which many existing studies had chosen, in order to investigate OA situations in a wider variety of articles including news articles or general articles. The rate of OA (26\%) was higher than the results in other studies. It implies that a gradual transition to $O A$ is occurring, and that the transition has been affected by turning-point occurrences for supporting OA movements from 2004 through 2005, such as the NIH public access policy and the governmental report or the new funding policy in England. Difference in the research method, however, is another factor that leads to the result. The method in this study is characterized as follows. One is that we used PubMed which indexed more kinds of journals than 'Web of Science' which was commonly used in many existing studies until now. Another is that we checked data by handwork instead of programming. The rate of $\mathrm{OA}$ in this study was probably raised by the characteristics of the method like this.

The most popular means of $O A$ is "OA or Hybrid OA journals" (72\%). The rate of OA through "authors' personal sites" or "institutional repositories" which marked high figure in existing researches was very low. The rate of articles published in journals with IF through "authors' personal sites" or "institutional repositories" is higher than that by other means. It could be possible to insist that researchers as authors would select only the articles published in prestigious journals, and make them OA. And yet the number of OA articles through "authors' personal sites" or "institutional repositories" was not large enough to assert that.

\section{International comparison}

The rates of $O A$ and the kinds of means of $O A$ varied from country to country, although it was a common trend in each country that the most popular means of OA was "OA or Hybrid OA journals". The rate of OA in the U.S. is quite high (30\%) but that in the U.K. is low, although both countries have advocated a policy sympathizing with $O A$. The situation of $O A$ in Japan may be affected by government policy (or the lack of it). While variable factors may cause the situation in which the rate of OA in Japan is lower than the average, 
Japanese policy which has not directly supported $O A$ is also responsible. Moreover, it is a distinguishing feature of Japan that $40 \%$ of OA articles were through J-STAGE supported by Japanese government. The purpose of J-STAGE, however, is to support academic societies in Japan to digitize their journals. It is only as a consequence that many Japanese society journals supported by J-STAGE provide their journal articles as OA articles.

This study totally showed that the situation of OA is complicated. Many other factors than those dealt with in this study may affect the situation of OA. For example, the policy (or position) of journals for $\mathrm{OA}$, the type of researchers' affiliation (university or private company), researchers' recognition for $\mathrm{OA}$ could be the factors, and further detailed investigation is necessary to know how these factors have worked.

The present study was funded in part by the Japan Society for the Promotion of Science (Grants-In-Aid for Scientific Research. Grant No.17500160).

\section{References}

Adachi, J., Negishi, M. Tutiya, S. Konishi, K., Oba, T. \& Okumura, S. (2003) Publishing role in the scholarly communication : Dispatch of Japanese scientific research seen in SPARC/JAPAN and university library (in Japanese) The Journal of Information Science and Technology Association 53(9), 429-434

Antelman, K. (2004) Do Open-Access Articles Have a Greater Research Impact? College and Research Libraries 65(5), 372-382. Retrieved February 6, 2006 from http://www.ala.org/ala/acrl/acrlpubs/crljournal/crl2004/crlseptember/antelman.pdf

Budapest Open Access Initiative (2002) Retrieved January 26, 2006, from http://www.soros.org/openaccess/read.shtml

Council for Science and Technology Policy (2005) Science and Technology Basic Plan (in Japanese) Retrieved February 2, 2006

from http://www8.cao.go.jp/cstp/output/toushin5.pdf

Directory of Open Access Journals Retrieved February 3, 2006 from http://www.doaj.org/

Hajjem, C., Harnad, S. and Gingras, Y. (2005) Ten-Year Cross-Disciplinary Comparison of the Growth of Open Access and How it Increases Research Citation Impact IEEE Data Engineering Bulletin 28(4), 39-47. Retrieved February 2, 2006

from http://sites.computer.org/debull/A05dec/hajjem.pdf

Harnad, S. (2005) The Implementation of the Berlin Declaration on Open Access : 
Report on the Berlin 3 Meeting Held 28 February - 1 March 2005 Southampton, UK D-Lib Magazine 11(3). Retrieved February 2, 2006

from http://www.dlib.org/dlib/march05/harnad/03harnad.html

Harnad, S., and Brody, T. (2004) Comparing the Impact of Open Access (OA)?vs. Non-OA Articles in the Same Journals D-Lib Magazine 10(6). Retrieved February 2, 2006 from http://www.dlib.org/dlib/june04/harnad/06harnad.html

House of Commons Science and Technology Committee (2004) Scientific Publications ; Free for all? Retrieved February 2, 2006

from http://www.publications.parliament.uk/pa/cm200304/cmselect/cmsctech/399/399.pdf

Kurtz, M.J., Eichihorn, G., Accomazzi, A., Grant, C., Demleitner, M., Henneken, E., Murray, S. S. (2005) The effect of use and access on citations Information Processing and Management 41(5), 1395-1402

Lawrence, S. (2001) Free online availability substantially increases a paper's impact Nature 411 (6837): 521. Retrieved February 2, 2006 from http://www.nature.com/nature/debates/e-access/Articles/lawrence.html

Miyairi, Y. (2005) Impact analysis on Open Access (in Japanese) Current

Awareness 284. Retrieved February 2, 2006

from http://www.ndl.go.jp/jp/library/current/no284/CA1559.html

Morris, S. (2006) When is a journal not a journal? A closer look at the DOAJ Learned Publishing 19(1), 73-76

Registry of Open Access Repositories Retrieved February 3, 2006 from http://archives.eprints.org/index.php

SPARC Open Access Newsletter, issue \#94. February 2, 2006 Retrieved February 6, 2006 from http://www.earlham.edu/ peters/fos/newsletter/02-02-06.htm

Suber, P. (2004) Open Access Overview Retrieved January 26, 2006 from http://www.earlham.edu/ peters/fos/overview.htm

Swan, A. and Brown, S. (2004) Open access self-archiving: An author study JISC Technical Report Retrieved February 6, 2006 from http://eprints.ecs.soton.ac.uk/10999/ 